\title{
Search for radial velocity and magnetic field pulsational variations in the roAp star $\gamma$ Equulei ${ }^{\star}$ (Research Note)
}

\author{
I. Savanov ${ }^{1,2}$, S. Hubrig ${ }^{3}$, G. Mathys ${ }^{3}$, A. Ritter ${ }^{1}$, and D. W. Kurtz ${ }^{4}$ \\ 1 Astrophysical Institute Potsdam (AIP), An der Sternwarte 16, 14482 Potsdam, Germany \\ e-mail: isavanov@aip.de \\ 2 Crimean Astrophysical Observatory, Nauchny, Crimea, Ukraine and Isaac Newton Institute of Chile, Crimean Branch \\ 3 European Southern Observatory, Casilla 19001, Santiago 19, Chile \\ ${ }^{4}$ Centre for Astrophysics, University of Central Lancashire, Preston PR1 1HE, UK
}

Received 22 September 2005 / Accepted 7 December 2005

\section{ABSTRACT}

\begin{abstract}
Aims. A new continuous $3.5 \mathrm{~h}$ series of high-resolution spectroscopic observations of the roAp star $\gamma$ Equ, with about 12 points per pulsation cycle, is presented to study the mean magnetic field modulus variability with the pulsation period.

Methods. Radial velocity variations with amplitudes of up to approximately $400 \mathrm{~m} \mathrm{~s}^{-1}$ are clearly detected in the rare earth lines present in the covered spectral range. Two frequencies are resolved in their analysis, which are consistent with frequencies previously observed in the star. No significant radial velocity variation is detected for the line Fe II $\lambda$ 6149.2. The mean magnetic field modulus derived from measurement of the wavelength separation of the magnetically resolved components of this line does not show any variation, with a $3 \sigma$ upper limit of $\sim 10 \mathrm{G}$. Results. While our results are consistent with the conclusion of the previous studies, difficulties to reconcile this result with those of other works may be attributed, in part at least, to the use of different diagnostic lines in different works and to the failure of all studies so far to fully resolve all the pulsation frequencies of $\gamma$ Equ.
\end{abstract}

Key words. stars: magnetic fields - stars: oscillations - stars: chemically peculiar

\section{Introduction}

The rapidly oscillating Ap (roAp) stars are chemically peculiar A stars that pulsate in high overtone acoustic modes with periods in the range 6-21 min. They have been studied intensively through photometry since their discovery (Kurtz 1982). Analysis of their light curves has provided a wealth of asteroseismic information, e.g. on the degrees of the pulsation modes and their distortion from normal modes, and on the luminosities of the studied stars. While photometry has been successful for discovering roAp stars and studying their frequency spectra, it is now clear that high-resolution spectroscopy is a superior tool for these purposes, and for other purposes out of the reach of photometry. In particular, high-resolution spectroscopic results show the short wavelength of the pulsation modes and, although the interpretation is complicated by the abnormal atmospheric structure of the atmospheres. they suggest vertical stratification of some ions, in particular the rare earths.

There is a close connection between the pulsations of roAp stars and their strong, large-scale organized magnetic fields.

^ Based on observations performed at the European Southern Observatory, La Silla, Chile (ESO Proposal No. 072.D-0375).
The oblique pulsator model (Kurtz 1982), in which the pulsation and magnetic axes of the star are aligned, has proved very successful for the interpretation of many observational properties; it certainly represents a very good first-order approximation of the geometrical structure of the pulsation. From the theoretical point of view, magnetic fields undoubtedly play a fundamental rôle in the excitation and selection mechanisms of the pulsation modes (e.g., Cunha \& Gough 2000; Balmforth et al. 2001; Bigot et al. 2000; Bigot \& Dziembowski 2002, 2003; Saio \& Gautschy 2004; Saio 2005).

Conversely, the effect of pulsation on the observed magnetic field remains to be established. Hubrig et al. (2004) have presented theoretical arguments suggesting that magnetic field variability with the pulsation period might be observable in roAp stars. From the observational point of view, the issue is hotly debated. Attempts to detect variations of the mean longitudinal magnetic field (i.e., the line-intensity weighted average over the visible stellar hemisphere of the component of the magnetic field vector along the line of sight) yielded apparently inconsistent results. Hubrig et al. (2004), applying the method introduced by Bagnulo et al. (2002) to diagnose the field from low-resolution spectropolarimetric observations with FORS1 


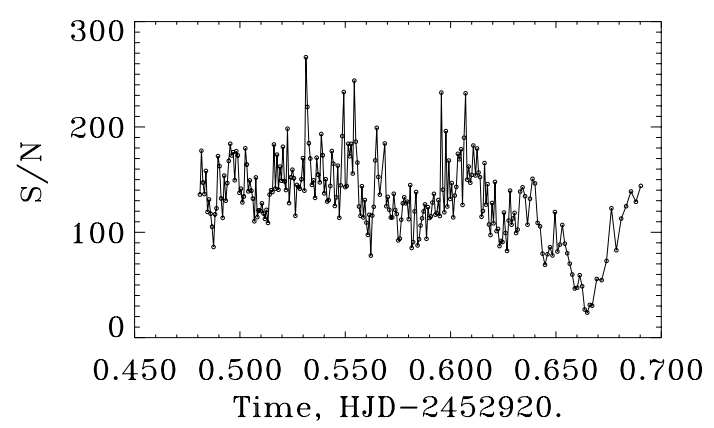

Fig. 1. $\mathrm{S} / \mathrm{N}$ ratio of the individual spectra.

at the VLT, did not find any variation at a level of the order of $100 \mathrm{G}$ over the pulsation cycle of six roAp stars. This sample includes $\gamma$ Equ, for which Leone \& Kurtz (2003) report the discovery in Nd III lines of longitudinal field variations with amplitudes of 110-240 $\mathrm{G}$ and a period of $12.1 \mathrm{~min}$. This discovery was subsequently questioned by Kochukhov et al. (2004a), who obtained an upper limit of 40-60 G for variations with the pulsation period in $13 \mathrm{Nd}$ III lines. Bychkov et al. (2005), using Balmer line photopolarimetry, also fail to detect any variation of the longitudinal field of $\gamma$ Equ with its pulsation period. On the other hand, in attempts to detect variations of the mean magnetic field modulus (that is, the lineintensity weighted average over the visible stellar hemisphere of the modulus of the magnetic vector) of the same star over its pulsation cycle, different authors also obtained different results. In an analysis based on the single line Fe II $\lambda 6149.2$, Savanov et al. (2003) reported the marginal detection of a variation of amplitude $99 \pm 53 \mathrm{G}$ with the pulsation period. However observation of other Fe II lines by Kochukhov et al. (2004b) set upper limits of 5-10 $\mathrm{G}$ for such variations. On the other hand, in the star HD $166473\left(=\mathrm{CoD}-37^{\circ} 12303\right)$, the analysis of the Zeeman triplet Eu II $\lambda 6437$ provided a tantalising hint at the occurrence of variations of the field modulus with the pulsation frequency, with an amplitude of $21 \pm 5 \mathrm{G}$ (Mathys et al. 2005a).

Here we present the results of a new attempt at detecting mean magnetic field modulus variations in $\gamma$ Equ (=HD 201601). At $V=4.7$, this star is one of the brightest known roAp stars.

\section{Observations and data reduction}

New observations of $\gamma$ Equ were obtained during the night of October 7-8, 2003, using the CES instrument at the ESO $3.6 \mathrm{~m}$ telescope; the detector was CCD \#61. The high-resolution image slicer was used, yielding a resolving power $\lambda / \Delta \lambda \sim$ 220000 . The spectra cover the wavelength range 6129-6169 $\AA$, which contains the strong rare earth lines Nd III $\lambda 6145.1$ and PrIII $\lambda 6160.2$ lines, as well as the Zeeman doublet Fe II $\lambda 6149.2$, which is the most frequently used field modulus diagnostic line (e.g., Mathys et al. 1997). This line is resolved into its magnetically split components in the spectra analysed in this work. In order to maximise the achievable $\mathrm{S} / \mathrm{N}$ ratio per spectral pixel and to minimise the CCD readout time overhead, the observations were performed with an on-chip binning factor of 4 in the direction perpendicular to spectral dispersion.
A total of 249 spectra were recorded over a continuous time interval of more than $5 \mathrm{~h}$. For the first 209 spectra the exposure time was $40 \mathrm{~s}$; with the overheads due to instrument setup and $\mathrm{CCD}$ readout, this corresponds to a duty cycle of $62 \mathrm{~s}$. After the first 110 spectra, thick cirrus started to pass across the sky, leading to significant degradation of the $\mathrm{S} / \mathrm{N}$ ratio of some spectra. Later on, a more uniform, rather dense absorption veil started to form, so that for spectra 210 to 239 , exposure time had to be increased to $80 \mathrm{~s}$ (duty cycle: $102 \mathrm{~s}$ ) to keep the $\mathrm{S} / \mathrm{N}$ ratio to usable values. Finally for the last 10 spectra, the exposure time was further increased to $180 \mathrm{~s}$ (close to the limit for adequate sampling of the pulsation cycle).

For purposes of wavelength calibration and monitoring of instrument stability, spectra of a ThAr arc lamp spectra were recorded just before the start, close to the middle, and just upon completion of the sequence of stellar exposures.

The spectra were reduced using standard IRAF and MIDAS routines. The $\mathrm{S} / \mathrm{N}$ ratio of the individual spectra (after reduction) is shown in Fig. 1. This ratio was estimated from consideration of spectral regions that appear to be reasonably free of lines, selected by spectrum synthesis. The rest of the analysis is restricted to the continuous series of the first 216 spectra (HJD 2452920.481 to 2452920.640 ), with $\mathrm{S} / \mathrm{N}$ values above 100 .

\section{Results}

The radial velocities (RVs) of the lines Nd III $\lambda 6145.1$ and Pr III $\lambda 6160.2$ were determined in the individual spectra by application of two different methods: line centre-of-gravity determination and Voigt profile fitting. Their variation with time is clearly seen in the top two panels ( $\mathrm{a}$ and $\mathrm{b}$ ) of Fig. 2. There is no significant difference between the behaviour derived through application of either RV measurement method. Amplitudes of up to approx. $400 \mathrm{~m} \mathrm{~s}^{-1}$ are observed; their modulation over a timescale of hours indicates that more than one frequency is present.

The corresponding amplitude spectra appear in the top two panels ( $a$ and $b$ ) of Fig. 3. They result from application to the data of Breger's (1990) code. Two peaks stand out, corresponding to periods of $11.58 \mathrm{~min}$ and $12.45 \mathrm{~min}$. The frequencies derived here are presented in Table 1 (with formal uncertainties corresponding to the half-width of the peaks); they are in good agreement with two of the four values obtained by Martinez et al. (1996) from the analysis of photometric data $(1.339,1.366,1.397$ and $1.427 \mathrm{mHz})$. The time span of our observations is insufficient to detect more frequencies that might possibly be present. We report also the phases of the variations in units of the period (their typical errors are in the region 0.04-0.08) with the zero point equal to HJD 2452920.4810.

The third panel (c) of Fig. 2 presents the radial velocity variations of an unidentified line at $6148.85 \AA$ which is a blend to the line Fe II $\lambda 6149.2$. The same two measurement methods as above were used. The pulsational behaviour of the line is typical of the REE lines. Figure 3c shows the corresponding amplitude spectra. 

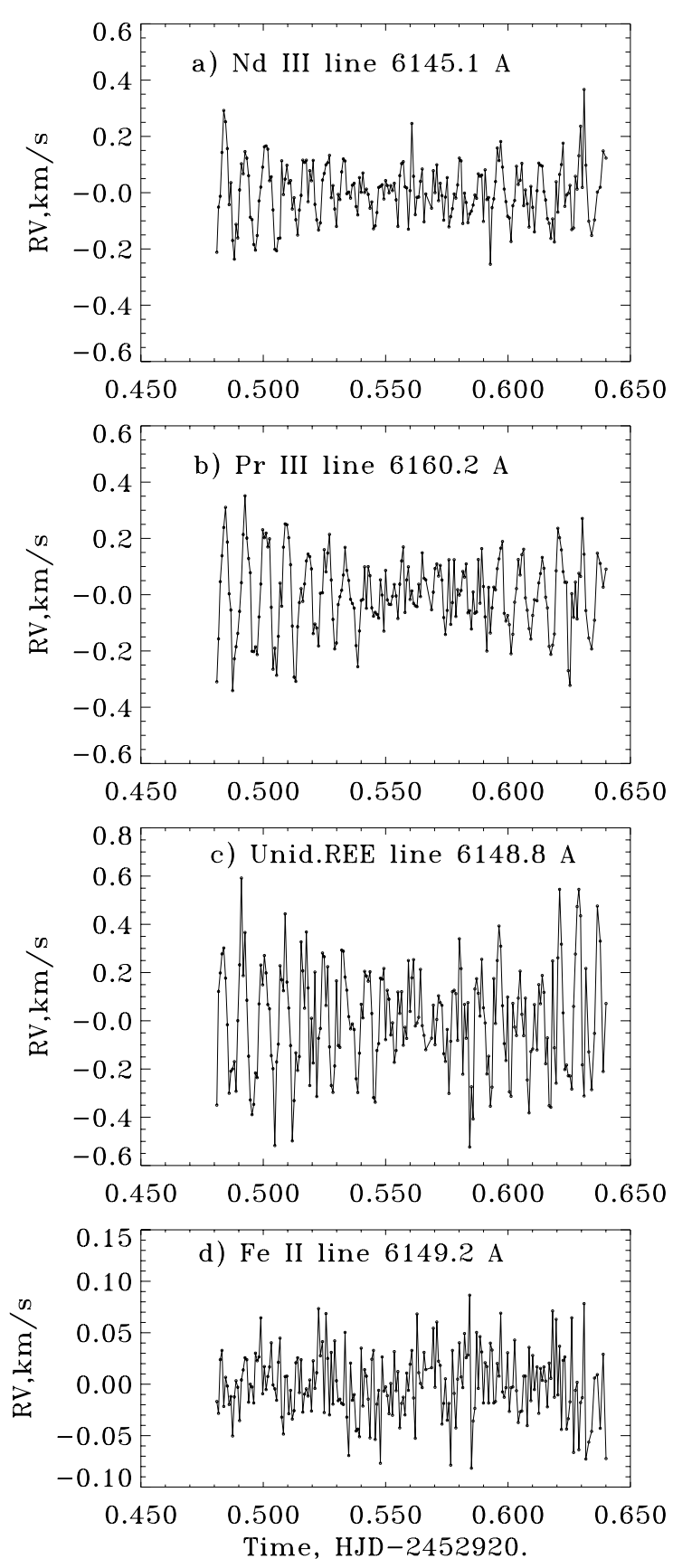

Fig. 2. Variations of the radial velocity of the lines $\mathrm{Nd} \operatorname{III} \lambda 6145.1$ a) and Pr III $\lambda 6160.2$ b), of the unidentified REE line $\lambda 6148.85$ c), and of the line Fe II $\lambda 6149.2$ d).

Finally in the bottom panels (d) of Figs. 2 and 3, the behaviour of the line Fe II $\lambda 6149.2$ is illustrated. In this case, the RV determinations were carried out by measurement of the centre of gravity of the line and by simultaneous fit of two Gaussians to its resolved components. No RV variation is detected for this line; the rms of the RV measurements is $32 \mathrm{~m} \mathrm{~s}^{-1}$. The highest-peak noise level in the amplitude spectrum is of the order of $15 \mathrm{~m} \mathrm{~s}^{-1}$.

We also measured, with the centre-of-gravity method, the difference $\left(\Delta \lambda_{\mathrm{c}}\right)$ in wavelength between the central parts of the two conponents of the line Fe II $\lambda 6149.2$ and derived the
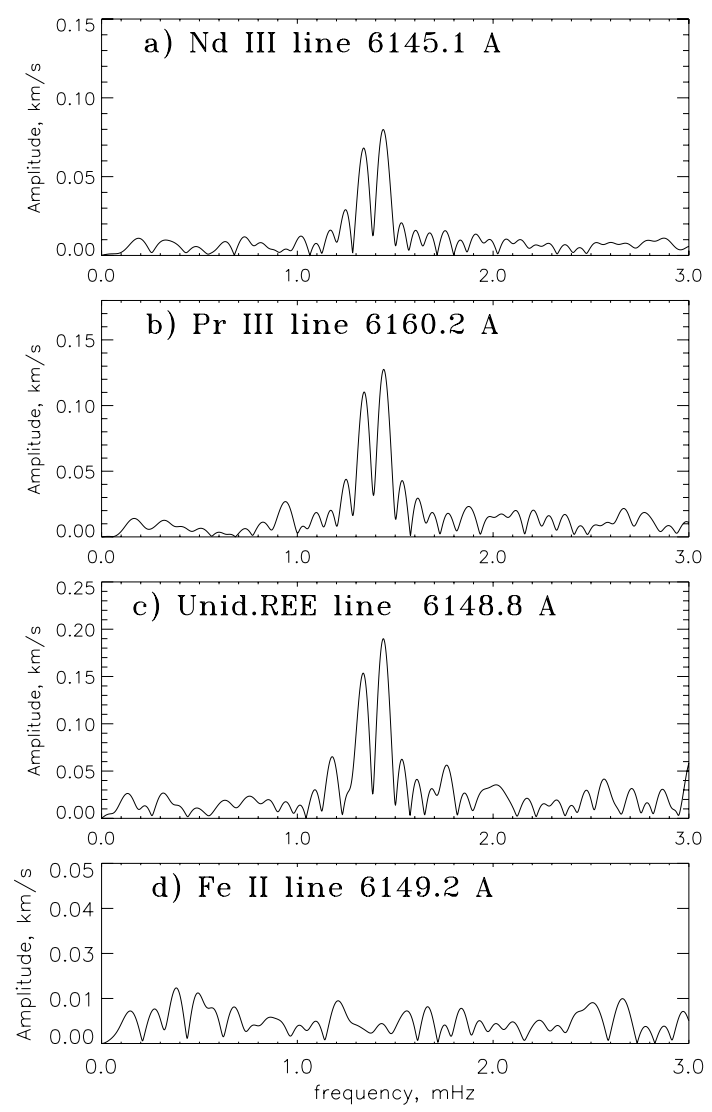

Fig. 3. Amplitude spectra for the radial velocity variations of the lines Nd III $\lambda 6145.1$ a) and PrIII $\lambda 6160.2$ b), of the unidentified REE line $\lambda 6148.85 \mathbf{c}$ ), and of the line Fe II $\lambda 6149.2 \mathbf{d}$ ).

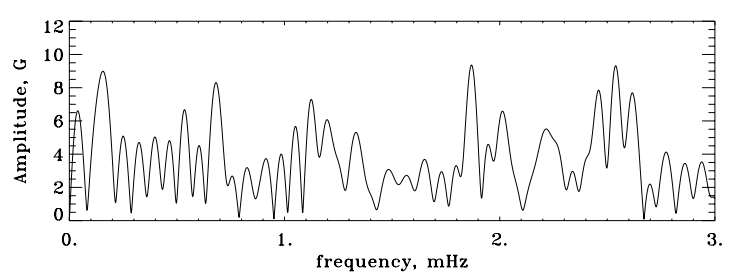

Fig. 4. Amplitude spectrum for the variations of magnetic field, determined from the line Fe II $\lambda$ 6149.2.

corresponding value of the mean magnetic field modulus, $H_{\mathrm{c}}$, by application of the relation

$H_{\mathrm{c}}=\Delta \lambda_{\mathrm{c}} /\left(g \Delta \lambda_{Z}\right)$,

where $g$ is the effective Landé factor of the transition, and $\Delta \lambda_{Z}$ is the Lorenz unit (see Mathys 1990 for details).

The average of all the values of $H_{\mathrm{c}}$ determined from the individual spectra is $3879 \pm 25 \mathrm{G}$. This is consistent with the view that the magnetic field modulus of $\gamma$ Equ is currently close to or slightly past the phase of its maximum, as suggested by Mathys et al. (1997, 2005b).

The amplitude spectrum of the individual mean field modulus measurements is presented in Fig. 4. No periodic variations are detected, with a highest-peak noise level of the order of $10 \mathrm{G}$. This is consistent with the noise level found for the $\mathrm{RV}$ of the whole line. As a result, an $3 \sigma$ upper limit of $\sim 10 \mathrm{G}$ can be set to any possible variations of the mean magnetic field 
Table 1. Frequency analysis of rare earth lines.

\begin{tabular}{lcccc}
\hline \multicolumn{1}{c}{ Line } & $v_{1}(\mathrm{mHz})$ & $v_{2}(\mathrm{mHz})$ & $\phi_{1}$ & $\phi_{2}$ \\
\hline Nd III $\lambda 6145.1$ & $1.339 \pm 0.034$ & $1.438 \pm 0.036$ & $0.97 \pm 0.06$ & $0.28 \pm 0.04$ \\
Pr III $\lambda 6160.2$ & $1.341 \pm 0.036$ & $1.442 \pm 0.037$ & $0.03 \pm 0.04$ & $0.42 \pm 0.06$ \\
Unid. $\lambda 6148.85$ & $1.337 \pm 0.036$ & $1.439 \pm 0.039$ & $0.75 \pm 0.08$ & $0.19 \pm 0.07$ \\
\hline
\end{tabular}

modulus of $\gamma$ Equ at the time of our observations, as diagnosed from the line Fe II $\lambda 6149.2$. This fully confirms the result of the similar study of Kochukhov et al. (2004b).

\section{Discussion}

Over the past few years, it has become clear that observations of different pulsation amplitudes (and possibly phases) in spectral lines of different ions, or even in different lines of a given ion, represent the common behaviour of an roAp star. This is interpreted as resulting from combination of the facts that the vertical wavelength of the pulsation modes is short with respect to the height of the line forming region, and that different lines arise from relatively thin layers at different atmospheric depths (probably in part due to vertical abundance stratification). Hence the pulsation characteristics sampled by a given line may differ considerably from those observable in another line.

Within this context, based on the observation of RV variability, it appears plausible that the line Fe II $\lambda 6149.2$ forms at a depth close to a pulsation node, while the rare earth lines discussed in this study form well away from such a node, at a higher level in the atmosphere.

The mechanism responsible for hypothetical variations of the magnetic field with the pulsation period obviously cannot be definitely established as long as it has not been possible to obtain reliable and sufficiently detailed observations of such variations. However one would intuitively expect the depth dependence of field variations to be to first order similar to that of RV variations. Within this framework, the non-detection of magnetic field variations in the present work may not be particularly surprising or especially constraining since the field was diagnosed from a Fe line that shows no RV variation, and hence must be formed close to a pulsation node.

Of course, the effect just described could in principle account for discrepant results between different attempts to detect magnetic field variations with the pulsation period in a given roAp star. However its consideration definitely does not resolve all of the current controversy. Indeed the detection of variability by Savanov et al. (2003) is based on analysis of Fe lines, just like the non-detections of Kochukhov et al. (2004b) and of the present study. Similarly, spectropolarimetric studies based on rare earth line analysis by Leone \& Kurtz (2003) and by Kochukhov et al. (2004a) also yield different results that cannot readily be explained in terms of depth dependence of the pulsation amplitude.

On the other hand, a complicating factor in $\gamma$ Equ is the presence of various pulsation modes with different frequencies; due to the resulting amplitude modulation ("frequency beating"), comparison of results based on data obtained at different epochs is not straightforward. The length of the time series analysed in this work is insufficient to resolve all the pulsation frequencies (Martinez et al. 1996; Savanov et al. 1999). The same is true for all other previous searches for magnetic field variations with the pulsation frequency in $\gamma$ Equ (see references in Sect. 1). Accordingly there is no way to identify unambiguously the pulsation modes observed in each study; most likely, the relative contributions of the various modes differ from study to study. This introduces an additional uncertainty factor in the interpretation of their results, and in the comparison between them.

In summary, future investigations of the possible variability of the magnetic fields of roAp stars as a result of their pulsation should be based on diagnostic lines formed far away from pulsation nodes, and they should be conducted in such a way as to ensure that the pulsation modes that are observed can be unambiguously identified.

\section{References}

Bagnulo, S., Szeifert, T., Wade, G. A., Landstreet, J. D., \& Mathys, G. 2002, A\&A, 389, 191

Balmforth, N. J., Cunha, M. S., Dolez, N., Gough, D. O., \& Vauclair, S. 2001, MNRAS, 323, 362

Bigot, L., \& Dziembowski, W. A. 2002, A\&A, 391, 235

Bigot, L., \& Dziembowski, W. A. 2003, Ap\&SS, 284, 217

Bigot, L., Provost, J., Berthomieu, G., Dziembowski, W. A., \& Goode, P. R. 2000, A\&A, 356, 218

Breger, M. 1990, Comm. Asteroseism., 6, 1

Bychkov, V. D., Bychkova, L. V., \& Madej, J. 2005, Acta Astron., 55, 141

Cunha, M. S., \& Gough, D. 2000, MNRAS, 319, 1020

Hubrig, S., Kurtz, D. W., Bagnulo, S., et al. 2004, A\&A, 415, 661

Kochukhov, O., Ryabchikova, T., \& Piskunov, N. 2004a, A\&A, 415, L13

Kochukhov, O., Ryabchikova, T., Landstreet, J. D., \& Weiss, W. W. 2004b, MNRAS, 351, L34

Kurtz, D. W. 1982, MNRAS, 200, 807

Leone, F., \& Kurtz, D. W. 2003, A\&A, 407, L67

Martinez, P., Weiss, W. W., Nelson, M. J., et al. 1996, MNRAS, 282, 243

Mathys, G. 1990, A\&A, 232, 151

Mathys, G., Hubrig, S., Landstreet, J. D., Lanz, T., \& Manfroid, J. 1997, A\&AS, 123, 353

Mathys, G., Kurtz, D. W., \& Elkin, V. G. 2005a, A\&A, submitted

Mathys, G., Manfroid, J., \& Wenderoth, E. 2005b, in preparation

Saio, H. 2005, MNRAS, 360, 1022

Saio, H., \& Gautschy, A. 2004, MNRAS, 350, 485

Savanov, I. S., Malanushenko, V. P., \& Ryabchikova, T. A. 1999, Astron. Let., 25, 802

Savanov, I., Musaev, F. A., \& Bondar, A. V. 2003, IBVS, 5468 